\title{
Enteropathogens in children less than 5 years of age with acute diarrhea: a 5-year surveillance study in the Southeast Coast of China
}

Shufa Zheng ${ }^{1,2}$, Fei Y $\mathbf{u}^{2}$, Xiao Chen ${ }^{1,2}$, Dawei Cui ${ }^{2}$, Yongzhang Cheng ${ }^{3}$, Guoliang Xie ${ }^{2}$, Xianzhi Yang ${ }^{2}$, Dongsheng Han ${ }^{4}$, Yiyin Wang ${ }^{2}$, Wen Zhang ${ }^{2}$ and Yu Chen ${ }^{1,2^{*}}$

\begin{abstract}
Background: Diarrhea is the second most common cause of death among children less than 5 years of age worldwide. The etiological agents of diarrhea in the southeast coastal area of China were studied from July 2009 to December 2014.

Methods: A total of the 2318 patients were enrolled in this study and examined for the presence of viruses, bacteria, and parasites. Multiplex real-time PCR was used for the detection of diarrheagenic Escherichia.coli (DEC). DEC strains were tested for susceptibility to a panel of 20 antibiotics using the Kirby-Bauer disc-diffusion method.

Results: Of the 2318 children with diarrhea, 962 (41.5\%) were positive for at least one pathogen. Rotavirus, human calicivirus (HucV), and DEC were predominant, with detection rates of $19.1 \%$ (443), $17.7 \%$ (411), and $7.6 \%$ (177), respectively. The prevalences of various pathogens in patients of different ages and in different seasons were not the same. The resistance rates of 177 strains of DEC to ampicillin, tetracycline, and cefazolin were $93.2 \%, 60.0 \%$, and $57.7 \%$, respectively.
\end{abstract}

Conclusions: Rotavirus, HuCV, and DEC were the main pathogens associated with diarrhea in Zhejiang, China. DEC possessed high levels of antibiotic resistance. Increased monitoring of etiological agents of diarrhea is necessary.

Keywords: Diarrhea, Enteropathogens, Children, Drug resistance

\section{Background}

Diarrhea is a maior cause of death in children under 5 years of age worldwide, with an estimated 1.5 million deaths per year. In developing countries, incidence rates range from 3.5 to 7.0 episodes per child per year during the first 2 years of life and from 2 to 5 episodes per child per year for the first 5 years of life [1]. Diarrhea is caused mainly by enteric pathogens, including viruses, bacteria,

\footnotetext{
*Correspondence: chenyu_zy@163.com

'State Key Laboratory for Diagnosis and Treatment of Infectious Diseases, Collaborative Innovation Center for Diagnosis and Treatment of Infectious Diseases, The First Affiliated Hospital, College of Medicine, Zhejiang University, No. 79, Qingchun Road, Hangzhou 310003, People's Republic China

${ }^{2}$ Key Laboratory of Clinical In Vitro Diagnostic Techniques of Zhejiang Province, No. 79, Qingchun Road, Hangzhou 310003, People's Republic China

Full list of author information is available at the end of the article
}

and parasites. There are relatively few comprehensive studies of the etiology of severe acute diarrhea in children admitted to the hospital. In China, an intensive survey of diarrheal diseases has not yet been performed, and the local characteristics of these diseases have thus been undefined. Therefore, the pathogen spectrum causing diarrhea requires investigation.

The People's Republic of China (PRC) is located in the eastern part of Asia, facing the Pacific Ocean on the east and encompassing temperate and tropical zones. Diarrhea is one of the main life-threatening diseases in china. During the survey period, the southeast coast of China had a population of approximately 54 million and a stable annual birth rate of 500,000. An epidemiological study of an infectious disease in a community is an initial step 
toward the introduction of the proper interventions for controlling the disease.

\section{Methods \\ Patients}

We collected samples and clinical information from 2532 patients. A total of 214 cases were eliminated because of nonconformance with the case definition or the presence of samples that were unqualified. The 2318 patients comprised all infants and children ( 0 to 5 years old) admitted directly to the enteric diseases clinic of the Children's Hospital, School of Medicine, Zhejiang University, from July 2009 to December 2014, with a diagnosis of acute diarrhea (defined as watery or loose stools $\geq 3$ times per day for $\leq 14$ days). The Children's Hospital is a large tertiary pediatric center serving the Zhejiang province.

\section{Microbiology}

Fresh fecal specimens were collected and divided into two aliquots. The first aliquot was sent to the clinical microbiology laboratory of the hospital. To identify the eggs of parasites, samples were concentrated, stained, and inoculated in different types of selective media and enrichment broths. Standard culture and identification methods were used to identify enteric pathogens. Isolated campylobacter was cultured in Columbia sheep blood AGAR inoculated with samples in a microaerophilic pot for $3-5$ days at $42{ }^{\circ} \mathrm{C}$. Five lactosefermenting colonies suggestive of Escherichia. coli from each MacConkey plate were selected and stored in semisolid media. The second aliquot was tested for the presence of viruses. The detection of group A rotaviruses was carried out with an enzyme-linked immunosorbent assay (ELISA) using a commercial kit (IDEIATM Rotavirus,
DAKO Ltd., UK) in accordance with the manufacturer's recommendations. Multiplex reverse transcriptionpolymerase chain reaction (RT-PCR) was carried out to detect the presence of the other viruses. The reverse transcription (RT) reaction was performed using random primers according to a previous report [2]. For multiplex PCR, two sets of specific primers were used (Table 1). The first primer set included equimolar amounts of specific primers for group B rotavirus, group C rotavirus and adenovirus. The second primer set included equimolar amounts of specific primers for norovirus GI, norovirus GII, sapovirus and astrovirus. The optimized reaction system consisted of $2.5 \mu \mathrm{l}$ of $10 \times$ Taq Buffer, $2.0 \mu \mathrm{l}$ of $25 \mathrm{mmol} / \mathrm{L} \mathrm{MgCl}_{2}, 2.0 \mu \mathrm{l}$ of $2.5 \mathrm{~mol} / \mathrm{L} \mathrm{dNTPs}, 0.125 \mu \mathrm{l}$ of $5 \mathrm{U} / \mu \mathrm{l}$ Taq DNA polymerase, $2.5 \mu \mathrm{l}$ of template cDNA/ DNA, $0.2 \mu \mathrm{l}$ of $33 \mu \mathrm{mol} / \mathrm{L}$ virus-specific primer and $\mathrm{ddH}_{2} \mathrm{O}$ to bring the total reaction volume to $25 \mu \mathrm{l}$ for each specimen. The cycling parameters were as follows: $94{ }^{\circ} \mathrm{C}$ for $5 \mathrm{~min}$ and 35 cycles of $94{ }^{\circ} \mathrm{C}$ for $30 \mathrm{~s}, 55^{\circ} \mathrm{C}$ for $30 \mathrm{~s}$, $72{ }^{\circ} \mathrm{C}$ for $1 \mathrm{~min}$; and $72{ }^{\circ} \mathrm{C}$ for $7 \mathrm{~min}$.

Characterization of diarrheagenic E. coli by multiplex PCR Multiplex real-time PCR was used for the detection of diarrheagenic E. coli (DEC). The target genes, primer sequences, PCR conditions, amplification parameters, and interpretation of results were performed according to a study by Muller et al. [3]. Strains that were $e s c V^{+} / b f p B{ }^{+}$were considered as typical enteropathogenic E. coli (EPEC), whereas $e s c V^{+} / b f p B^{-}$strains were identified as atypical EPEC. The quality control strains were EPEC CMCC44155 (esc $V:+$ ), enterohemorrhagic (or Shiga toxin-producing) $E$. coli (STEC) EDL933 (stx1: +; stx2: +; and escV: +), enterotoxigenic E. coli (ETEC) H10407 (elt: +; estIa: +; and estIb +), enteroinvasive E. coli (EIEC) CMCC44825

Table 1 Virus-specific primers used for the multiplex PCR

\begin{tabular}{|c|c|c|c|c|c|}
\hline Virus & Primer & Polarity & Sequence $\left(5^{\prime}-3^{\prime}\right)$ & Product size (bp) & Reference \\
\hline \multirow[t]{2}{*}{ Group B rotavirus } & B5-2 & + & GGCAATAAAATGGCTTCATTGC & 814 & [13] \\
\hline & B5-3 & - & GGGTITTACAGCTTCGGCT & & \\
\hline \multirow[t]{2}{*}{ GroupC rotavirus } & NG8S1 & + & ATTATGCTCAGACTATCGCCAC & 352 & [13] \\
\hline & NG8S2 & - & GTTTCTGTACTAGCTGGTGAAC & & \\
\hline \multirow[t]{2}{*}{ Adenovirus } & Ad1 & + & TTCCCCATGGCICAYAACAC & 482 & [13] \\
\hline & $\mathrm{Ad} 2$ & - & CCCTGGTAKCCRATRTTGTA & & \\
\hline \multirow[t]{2}{*}{ Norovirus (Gl) } & G1-SKF & + & CTGCCCGAATTYGTAAATGA & 330 & [14] \\
\hline & GI-SKR & - & CCAACCCARCCATTRTACA & & \\
\hline \multirow[t]{2}{*}{ Norovirus (GII) } & $\operatorname{CoG} 2 \mathrm{~F}$ & + & CARGARBCNATGTTYAGRTGGATGAG & 387 & [14] \\
\hline & G2-SKR & - & CCRCCNGCATRHCCRTTRTACAT & & \\
\hline \multirow[t]{2}{*}{ Sapovirus } & SLV-5317 & + & CTCGCCACCTACRAWGCBTGGTT & 434 & [14] \\
\hline & SLV-5749 & - & CGGRCYTCAAAVSTACCBCCCCA & & \\
\hline \multirow[t]{2}{*}{ Astrovirus } & PreCAP1 & + & GGACTGCAAAGCAGCTTCGTG & 719 & [14] \\
\hline & $82 \mathrm{~b}$ & - & GTGAGCCACCAGCCATCCCT & & \\
\hline
\end{tabular}


(invE: + ), and enteroaggregative E. coli (EAEC) O42 (astA: +; aggR: +; and pic: +).

\section{Antimicrobial susceptibility testing}

DEC strains were tested for susceptibility to a panel of 20 antibiotics with the Kirby-Bauer disc-diffusion method. Results were interpreted according to the guidelines of the Clinical Laboratory Standards Institute (CLSI, 2010). The definitions of multidrug resistance were obtained from the report of Magiorakos et al. [4]. Quality control strains contained E. coli ATCC 25922, Staphylococcus aureus ATCC 25923, and Klebsiella pneumoniae ATCC700603 (producing extended-spectrum beta-lactamase, ESBL).

\section{Data analysis}

Statistical analyses were performed using Statistical Package for Social Sciences (SPSS) version 17.0. The statistical significance of the differences between the groups was evaluated by the $x^{2}$-test or Fisher's exact test (whenever necessary). All $p$ values reported are two-sided. $p<0.05$ was considered to be statistically significant.

\section{Results}

From July 2009 to December 2014, a total of 2318 patients were enrolled in this study. The mean age of children with diarrhea was $1.17 \pm 1.23$ (SD) years. Among the patients, the ratio of males to females was 1.72:1. Basic information and clinical symptoms of patients analyzed are presented in Table 2.

Of the 2318 children with diarrhea, 962 (41.5\%) were positive for at least one pathogen. Pathogenic viruses were isolated from 728 (31.4\%) samples, bacterial were present in 285 (12.3\%) samples, and parasites were detected in $18(0.8 \%)$ samples. Rotavirus, human calicivirus $(\mathrm{HucV})$, and DEC were detected most often. The incidences of enteropathogens detected from the collected specimens are presented in Table 3.

Of the 2318 samples examined from patients with diarrhea, rotavirus was the most common pathogen with a prevalence of $19.1 \%$. The detection rate in children aged 1 to 2 years was $23.9 \%$, significantly higher than that in children in the other groups $(p<0.001)$. Rotavirus infection was most prevalent in children during the winter $(32.7 \%)$, but Was also present in children in the spring $(21.5 \%)$ and fall (16.7 \%), and the lowest infection rate was seen in the summer $(9.5 \%)$. There was a significant difference in rotavirus prevalence between seasons $(p<0.001)$.

$\mathrm{HucV}$ was the second most frequently identified enteric pathogen with a prevalence of $17.7 \%$. Of the samples positive for $\mathrm{HucV}, 320$ (13.8\%) were positive for norovirusII, $52(2.2 \%)$ were positive for norovirusI, and 39 (1.7 \%) were positive for sappovirus. HucV infection was most prevalent in children aged 2-5 years old
Table 2 Basic information and clinical symptoms of the 2318 children with acute diarrhea

\begin{tabular}{ll}
\hline Characteristic & No. of case (\%) \\
\hline Seasons & \\
Spring & $446(19.2)$ \\
Summer & $855(36.9)$ \\
Fall & $414(17.9)$ \\
Winter & $603(26.0)$ \\
Age (years) & \\
$<1$ & $1013(43.7)$ \\
1-2 & $711(30.7)$ \\
$3-5$ & $594(25.6)$ \\
Mean age \pm SD & $1.17 \pm 1.23$ \\
Sex ratio (M/F) & $1.72 / 1$ \\
Diarrhea & \\
Frequency & $4.17 \pm 2.43$ \\
Loose & $772(33.3)$ \\
Mucus & $248(10.7)$ \\
Watery & $361(15.6)$ \\
Bloody & $13(0.5)$ \\
Unknown & $924(39.9)$ \\
Fomiting & $781(32.5)$ \\
Wrequency & $2.28 \pm 1.71$ \\
Abtomach contents & $57(2.7)$ \\
\hline & $724(29.8)$ \\
Antibiotic consumption & $212(8.9)$ \\
& $147(6.0)$ \\
Fever & $114(4.4)$ \\
\hline
\end{tabular}

(23.1\%). The detection rate was highest in the spring $(22.6 \%)$. There were significantly differences in infection rates in different seasons $(p<0.001)$.

The third most common pathogen in diarrheal samples was DEC, the most prevalent bacterial enteropathogen, with an isolation rate of $7.6 \%$. Of the samples positive for DEC, 104 (4.5\%) were positive for EAEC, 40 (1.7 \%) for EPEC, 24(1.0 \%) for ETEC, 7 (0.3 \%) for EHEC, and $2(0.1 \%)$ for EIEC. The highest detection rate was found in the 2- to 5-year-old age group $(12.3 \%)$, and there were statistically significant differences between age groups $(p<0.001)$. EAEC, EPEC, and ETEC were more frequently isolated in children more than two years of age (7.2\% $3.2 \%$ and $1.9 \%$, respectively), whereas EIEC and ETEC were less frequently found. The detection rate in summer $(11.5 \%)$ was higher than that in the other seasons $(p<0.001)$. No children were identified with infections of more than two DEC.

In addition, we detected adenovirus (2.2\%), astrovirus (1.6 \%), Shigella spp. (1.5\%), Vibrio parahaemolyticus 
Table 3 Incidence and age distribution of enteropathogens isolated from patients

\begin{tabular}{|c|c|c|c|c|c|c|c|c|c|c|}
\hline \multirow[t]{2}{*}{ Pathogen } & \multirow{2}{*}{$\begin{array}{l}\text { No. (\%) of } \\
\text { isolates in } \\
\text { patients }\end{array}$} & \multicolumn{3}{|c|}{$\begin{array}{l}\text { No. (\%) of isolates in patients of the } \\
\text { following ages }\end{array}$} & \multirow[t]{2}{*}{$p$ value } & \multicolumn{4}{|c|}{$\begin{array}{l}\text { No. (\%) of isolates in patients of the following } \\
\text { seasons }\end{array}$} & \multirow[t]{2}{*}{$p$ value } \\
\hline & & $\begin{array}{l}<1 \text { year } \\
(n=1013)\end{array}$ & $\begin{array}{l}1-2 \text { years } \\
(n=711)\end{array}$ & $\begin{array}{l}2-5 \text { years } \\
(n=594)\end{array}$ & & $\begin{array}{l}\text { Spring } \\
(n=446)\end{array}$ & $\begin{array}{l}\text { Summer } \\
(n=855)\end{array}$ & $\begin{array}{l}\text { Fall } \\
(n=414)\end{array}$ & $\begin{array}{l}\text { Winter } \\
(n=603)\end{array}$ & \\
\hline Rotavirus & $443(19.1)$ & 184(18.2) & $170(23.9)$ & 89(15.0) & 0.000 & $96(21.5)$ & $81(9.5)$ & $69(16.7)$ & 197(32.7) & 0.000 \\
\hline Calicivirus & $411(17.7)$ & 151(14.9) & 123(17.3) & 137(23.1) & 0.000 & $101(22.6)$ & 130(15.2) & $61(14.7)$ & 119(19.7) & 0.002 \\
\hline Norovirus I & $52(2.2)$ & $14(1.4)$ & $15(2.1)$ & 23(3.9) & 0.005 & $4(0.9)$ & $18(2.1)$ & $16(3.9)$ & $14(2.3)$ & 0.033 \\
\hline Norovirus II & $320(13.8)$ & $127(12.5)$ & 93(13.1) & $100(16.8)$ & 0.044 & $90(20.2)$ & 101(11.8) & $41(9.9)$ & 88(14.6) & 0.000 \\
\hline Sappovirus & $39(1.7)$ & $10(1.0)$ & $15(2.1)$ & $14(2.4)$ & 0.068 & $7(1.6)$ & $11(1.3)$ & $4(1.0)$ & $17(2.8)$ & 0.077 \\
\hline Astrovirus & $38(1.6)$ & $13(1.3)$ & $12(1.7)$ & $13(2.2)$ & 0.383 & $9(2.0)$ & $9(1.1)$ & $5(1.2)$ & $15(2.5)$ & 0.145 \\
\hline Adenovirus & $51(2.2)$ & $22(2.2)$ & $24(3.4)$ & $5(0.8)$ & 0.008 & $9(2.0)$ & $11(1.3)$ & $14(3.4)$ & $17(2.8)$ & 0.067 \\
\hline Diarrheagenic E. coli & $177(7.6)$ & $6(6.0)$ & $43(6.0)$ & 73(12.3) & 0.000 & $24(5.4)$ & $98(11.5)$ & $21(5.1)$ & $34(5.6)$ & 0.000 \\
\hline EAEC & 104(4.5) & $39(3.8)$ & $22(3.1)$ & $43(7.2)$ & 0.001 & $14(3.1)$ & $62(7.3)$ & $7(1.7)$ & $21(3.5)$ & 0.000 \\
\hline EPEC & $40(1.7)$ & $8(0.8)$ & $13(1.8)$ & 19(3.2) & 0.002 & $5(1.1)$ & $19(2.2)$ & $9(2.2)$ & $7(1.2)$ & 0.281 \\
\hline ETEC & $24(1.0)$ & $7(0.7)$ & $6(0.8)$ & $11(1.9)$ & 0.071 & $5(1.1)$ & $11(1.3)$ & $2(0.5)$ & $6(1.0)$ & 0.651 \\
\hline EHEC & $7(0.3)$ & $5(0.5)$ & $2(0.3)$ & $0(0)$ & NA & $0(0)$ & $4(0.5)$ & $3(0.7)$ & $0(0)$ & NA \\
\hline EIEC & $2(0.1)$ & $2(0.2)$ & 0 0) & $00)$ & NA & 0 0) & $20.2)$ & 0 0) & $O(0)$ & NA \\
\hline Shigella spp. & $34(1.5)$ & $15(1.5)$ & $12(1.7)$ & $7(1.2)$ & 0.747 & $4(0.9)$ & $21(2.5)$ & $7(1.7)$ & $2(0.3)$ & 0.006 \\
\hline S. flexneri & $2(0.1)$ & $0(0)$ & $0(0)$ & $2(0.3)$ & NA & $0(0)$ & $2(0.2)$ & $0(0)$ & $0(0)$ & NA \\
\hline S. sonnei & $26(1.1)$ & $11(1.1)$ & $10(1.4)$ & $5(0.8)$ & 0.621 & $4(0.9)$ & $18(2.1)$ & $3(0.7)$ & $1(0.2)$ & 0.004 \\
\hline S. boydii & $6(0.3)$ & $4(0.4)$ & $2(0.3)$ & $0(0)$ & NA & $0(0)$ & $1(0.1)$ & $4(1.0)$ & $1(0.2)$ & NA \\
\hline Vibrio parahaemolyticus & $29(1.3)$ & $10(1.0)$ & $6(0.8)$ & $13(2.2)$ & 0.056 & $2(0.4)$ & 16(1.9) & $7(1.7)$ & $4(0.7)$ & 0.062 \\
\hline Salmonella spp. & $15(0.6)$ & $4(0.4)$ & $2(0.3)$ & $9(1.5)$ & 0.009 & $2(0.4)$ & $9(1.1)$ & $2(0.5)$ & $2(0.3)$ & 0.312 \\
\hline Campylobacter jejuni & $10(0.4)$ & $5(0.5)$ & $2(0.3)$ & $3(0.5)$ & 0.764 & $0(0)$ & $5(0.6)$ & $3(0.7)$ & $2(0.3)$ & NA \\
\hline Plesiomonas shigelloides & $9(0.4)$ & $2(0.2)$ & $2(0.3)$ & $5(0.8)$ & 0.115 & $2(0.4)$ & $5(0.6)$ & $2(0.5)$ & $0(0)$ & NA \\
\hline Vibrio cholerae & $6(0.3)$ & $4(0.4)$ & $0(0)$ & $2(0.3)$ & NA & $0(0)$ & $3(0.4)$ & $0(0)$ & $3(0.5)$ & NA \\
\hline Aeromonas hydrophila & $5(0.2)$ & $2(0.2)$ & $0(0)$ & $3(0.5)$ & NA & $0(0)$ & $5(0.6)$ & $0(0)$ & $0(0)$ & NA \\
\hline Yersinia enterocolitica & $4(0.2)$ & $5(0.5)$ & $0(0)$ & $0(0)$ & NA & $1(0.2)$ & $3(0.4)$ & $0(0)$ & $0(0)$ & NA \\
\hline Campylobacter spp. & $3(0.1)$ & $1(0.1)$ & $0(0)$ & $2(0.3)$ & NA & $0(0)$ & $0(0)$ & $2(0.5)$ & $1(0.2)$ & NA \\
\hline Amoeba & $14(0.6)$ & $1(0.1)$ & $2(0.3)$ & $10(1.7)$ & 0.000 & $2(0.4)$ & $8(0.9)$ & $4(1.0)$ & $0(0)$ & NA \\
\hline Giardia lamblia & $2(0.1)$ & $0(0)$ & $2(0.3)$ & $0(0)$ & NA & $0(0)$ & $2(0.2)$ & $0(0)$ & $0(0)$ & NA \\
\hline Cryptosporidiosis & $2(0.1)$ & $0(0)$ & $0(0)$ & $2(0.3)$ & NA & $0(0)$ & $0(0)$ & $2(0.5)$ & $0(0)$ & NA \\
\hline
\end{tabular}

$\mathrm{NA}$, not applicable; Boldface, indicates statistical significance $(\mathrm{p}<0.05)$

(1.3\%), Salmonella spp. (0.6 \%), Campylobacter jejuni (0.4\%), Plesiomonas shigelloides (0.4\%), Vibrio cholerae (0.3\%), Aeromonas hydrophila (0.2 \%), Yersinia enterocolitica (0.2 \%), Campylobacter spp. (0.1\%), Amoeba (0.6\%), Giardia lamblia (0.1\%), and Cryptosporidiosis $(0.1 \%)$ in samples. The detection rates for participants of different ages and during different seasons are shown in Table 3.

The occurrences of mixed infections with enteric pathogens is shown in Table 4. Among children with diarrhea, $247(10.7 \%)$ were infected with two or more pathogens. The most frequent pattern of mixed infection was the combination of rotavirus with $\mathrm{HucV}$ (121 patients). In total, 192 specimens exhibited mixed infections associated with rotavirus, 182 specimens exhibited mixed infections associated with $\mathrm{HucV}$, and 58 specimens exhibited mixed infections associated with DEC.

The antimicrobial resistance patterns of the DEC, expressed as a percentage of isolates are shown in Table 5 . The resistance rates of 177 strains to ampicillin, tetracycline, and cefazolin were 93.2, 60.0, and $57.7 \%$, respectively. The resistance rates to cefepime, cefoxitin, piperacillin-tazobactam, and cefoperazone-sulbactam were all below $10 \%$. No resistance to imipenem or meropenem was detected. The antimicrobial resistance rates in EAEC, ETEC, and EPEC were no significantly different. 
Table 4 Children with multiple pathogens isolated from a single specimen in diarrhea children

\begin{tabular}{ll}
\hline Patterns of infection & No. of case (\%) \\
\hline Rotavirus + Calicivirus & $121(5.2)$ \\
Rotavirus + Calicivirus + Diarrheagenic E. coli & $21(0.9)$ \\
Rotavirus + Diarrheagenic E. coli & $19(0.8)$ \\
Calicivirus + Diarrheagenic E. coli & $14(0.6)$ \\
Rotavirus + Adenovirus & $12(0.5)$ \\
Rotavirus + Astrovirus & $11(0.5)$ \\
Rotavirus + Calicivirus + Adenovirus & $9(0.4)$ \\
Calicivirus + Adenovirus & $7(0.3)$ \\
Calicivirus + Astrovirus & $7(0.3)$ \\
Rotavirus + Calicivirus + Astrovirus & $5(0.2)$ \\
Rotavirus + Vibrio parahaemolyticus & $3(0.1)$ \\
Calicivirus + Salmonella spp. & $3(0.1)$ \\
Calicivirus + Shigella spp. & $2(0.1)$ \\
Salmonella spp. + Vibrio cholerae & $2(0.1)$ \\
Calicivirus + Vibrio parahaemolyticus & $2(0.1)$ \\
Salmonella spp. + Yersinia intestinal colon & $1(0.0)$ \\
Vibrio parahaemolyticus + Vibrio cholerae & $1(0.0)$ \\
Rotavirus + Calicivirus + Astrovirus + Adenovirus & $1(0.0)$ \\
Calicivirus + Adenovirus + Shigella spp. & $1(0.0)$ \\
Diarrheagenic E. coli + Campylobacter jejuni + Yersinia & $1(0.0)$ \\
enterocolitica & \\
Rotavirus + Adenovirus + Diarrheagenic E. coli & $1(0.0)$ \\
Calicivirus + Diarrheagenic E. coli + Campylobacter jejuni & $1(0.0)$ \\
Rotavirus + Calicivirus + Yersinia enterocolitica & $1(0.0)$ \\
Rotavirus + Calicivirus + Astrovirus + Diarrheagenic E. coli & $1(0.0)$ \\
\hline &
\end{tabular}

\section{Discussion}

The State Key Laboratory for diagnosis and treatment of infectious disease at Zhejiang University is an important component of the infectious surveillance platform in China, and it is responsible for monitoring the spectrum of pathogens in Zhejiang Province and the surrounding areas. Monitoring the rates of diarrhea is an important aspect of monitoring and has always been our top priority. In the current study, at least one pathogen was isolated from $41.5 \%(962 / 2318)$ of stool samples from patients with diarrhea. This percentage is lower than that in previous studies carried out in developing countries addressing the presence of virus, bacteria or parasites $[5,6]$. Various factors may account for such a difference. Many studies have shown that diarrhea prevalence is strongly associated with sanitation [7-9]. Zhejiang Province located on the southeastern coast of China, is on the forefront of reform. Living standards and health conditions have greatly improved in recent years, which is the main reason for the reduction in the prevalence of diarrhea pathogens. In addition, we found that the Proteus detection rate in the region was up to $15 \%$, but the pathogenicity of Proteus is considered controversial [10]. Finally, antimicrobial therapy has been initiated prior to sample collection in some cases, reducing the percentage of bacterial enteropathogens isolated.

Several studies have documented the prevalence of rotavirus in children with acute gastroenteritis to be in the range of 13 to $58 \%$ [1, 11-14]. The overall detection rate of rotavirus among children aged $<5$ years was $19.1 \%$, which was significantly lower than rates reported in surveys conducted in many other countries in Asia, Europe, and the Americas $[11,13,15,16]$. The main reason for this discrepancy could be sample selection bias. In the current study, the detection rate during winter was as high as $32.8 \%$, but the specimens collected in winter only accounted for $1 / 4$ of all the specimens, thereby leading to a reduction in the overall detection rate. We found that the detection rate of rotavirus was higher in children under the age of 3 than in children of other ages and that it was higher in winter than in other seasons, which is consistent with other reports $[15,17,18]$. Prevention and control of rotavirus infections during winter should be emphasized, especially for infants and young children under 3 years of age. However, interestingly, other pathogens were detected in approximately $35 \%$ of rotavirus-positive samples. Although other surveillance studies reported that rotavirus could be the putative cause of diarrhea, we would like to determine that whether rotavirus was truly the etiological agent of disease in these patients, as well as the contribution of the other pathogens to the disease process.

This survey confirms worldwide studies identifying $\mathrm{HucV}$ as the second most common cause of severe diarrhea in young children admitted to hospitals in developed and developing countries [19-21]. Our surveillance data indicated that $\mathrm{HucV}$ was responsible for $17.7 \%$ of diarrhea cases in Zhejiang. Fang et al. reported that from 1999 to 2005, the HucV detection rate in Zhejiang region was $38.6 \%$ [22]. Data in our study, as well as in previous studies, suggest that $\mathrm{HucV}$ is a common viral agent associated with childhood diarrhea in Zhejiang. The variation in detection rates is most likely related to the detection methods as well as the target subjects. In all cases of $\mathrm{HucV}$ infection, norovirus was present in approximately $90.4 \%$ of cases, which was observed in Lanzhou City, China [16]. Norovirus is believed to be a major pathogen leading to diarrheal outbreak and causes symptomatic infections in older children and adults [15]. However, in the current study, we found that the detection rate of norovirus among participants younger than 3 years was lower than that among participants older than 3 years, therefore, younger children infected with 
Table 5 Antimicrobial resistance patterns of Diarrheagenic Escherichia coli (DEC) isolates

\begin{tabular}{|c|c|c|c|c|c|c|c|c|c|c|c|}
\hline \multirow[t]{2}{*}{ Antimicrobial } & \multicolumn{2}{|c|}{$\begin{array}{l}\text { DEC } \\
(n=177)\end{array}$} & \multicolumn{2}{|c|}{$\begin{array}{l}\text { EAEC } \\
(n=104)\end{array}$} & \multicolumn{2}{|c|}{$\begin{array}{l}\text { ETEC } \\
(n=40)\end{array}$} & \multicolumn{2}{|c|}{$\begin{array}{l}\text { EPEC } \\
(n=24)\end{array}$} & \multicolumn{2}{|c|}{$\begin{array}{l}\text { STEC } \\
(n=7)\end{array}$} & \multirow[t]{2}{*}{$p^{a}$} \\
\hline & $\% \mathrm{R}$ & $\% \mathrm{~S}$ & $\% \mathrm{R}$ & $\% \mathrm{~S}$ & $\% \mathrm{R}$ & $\% \mathrm{~S}$ & $\% \mathrm{R}$ & $\% \mathrm{~S}$ & $\% R$ & $\% \mathrm{~S}$ & \\
\hline AMP & 93.2 & 2.8 & 96.2 & 1.9 & 95.0 & 0 & 83.3 & 4.2 & 71.4 & 28.6 & 0.055 \\
\hline TCY & 60.0 & 38.3 & 61.5 & 38.5 & 55.0 & 37.5 & 79.2 & 20.8 & 0 & 100 & 0.258 \\
\hline $\mathrm{CZO}$ & 57.7 & 26.9 & 55.8 & 27.9 & 55.0 & 27.5 & 79.2 & 20.8 & 28.6 & 28.6 & 0.093 \\
\hline SXT & 57.2 & 39.5 & 63.5 & 33.7 & 55.0 & 37.5 & 50.0 & 50.0 & 0 & 100 & 0.467 \\
\hline PIP & 51.4 & 32.5 & 49.0 & 31.7 & 55.0 & 27.5 & 62.5 & 33.3 & 28.6 & 71.4 & 0.209 \\
\hline CXM & 46.3 & 50.3 & 51.0 & 49.0 & 37.5 & 55.0 & 45.8 & 54.2 & 28.6 & 28.6 & 0.347 \\
\hline SAM & 45.1 & 37.1 & 42.3 & 36.5 & 55.0 & 37.5 & 45.8 & 29.2 & 28.6 & 71.4 & 0.392 \\
\hline CTX & 42.3 & 54.9 & 49.0 & 51.0 & 27.5 & 62.5 & 41.7 & 54.2 & 28.6 & 71.4 & 0.064 \\
\hline GEN & 35.4 & 60.0 & 36.5 & 59.6 & 37.5 & 55.0 & 37.5 & 58.3 & 0 & 100 & 0.992 \\
\hline ATM & 21.7 & 68.6 & 27.9 & 63.5 & 10.0 & 90.0 & 20.8 & 54.2 & 0 & 71.4 & 0.070 \\
\hline CIP & 17.7 & 79.4 & 23.1 & 72.1 & 7.5 & 92.5 & 16.7 & 83.3 & 0 & 100 & 0.095 \\
\hline CAZ & 14.8 & 85.2 & 19.2 & 80.8 & 10.0 & 90.0 & 8.3 & 91.7 & 0 & 100 & 0.226 \\
\hline AMC & 12.0 & 69.1 & 14.4 & 72.1 & 10.0 & 62.5 & 0.0 & 66.7 & 28.6 & 71.4 & 0.127 \\
\hline FEP & 11.4 & 76.5 & 14.4 & 74.0 & 10.0 & 72.5 & 4.2 & 95.8 & 0 & 71.4 & 0.343 \\
\hline FOX & 5.1 & 93.7 & 6.7 & 91.3 & 0 & 100 & 0 & 100 & 28.6 & 71.4 & 0.106 \\
\hline TZP & 4.0 & 90.9 & 6.7 & 85.6 & 0 & 100 & 0 & 95.8 & 0 & 100 & 0.106 \\
\hline CSL & 2.3 & 74.3 & 3.8 & 72.1 & 0 & 72.5 & 0 & 79.2 & 0 & 100 & 0.283 \\
\hline AMK & 1.1 & 93.7 & 1.9 & 93.3 & 0 & 90.0 & 0 & 100 & 0 & 100 & 0.536 \\
\hline IPM & 0 & 100 & 0 & 100 & 0 & 100 & 0 & 100 & 0 & 100 & $N A^{b}$ \\
\hline MEM & 0 & 100 & 0 & 100 & 0 & 100 & 0 & 100 & 0 & 100 & $N A^{b}$ \\
\hline
\end{tabular}

EAEC enteroaggregative $E$. coli, ETEC enterotoxigenic $E$. coli, EPEC enteropathogenic $E$. coli, STEC Shiga toxin-producing $E$. coli, R Resistance, S susceptibility, AMP Ampicillin, TCY Tetracycline, CZO Cefazolin, SXT Trimethoprim-Sulfamethoxazole, PIP Piperacillin, CXM Cefuroxime, SAM Ampicillin-Sulbactam, CTX Cefotaxime, GEN Gentamicin, ATM Aztreonam, CIP Ciprofloxacin, CAZ Ceftazidime, AMC Amoxicillin-Clavulanicacid, FEP Cefepime, FOX Cefoxitin, TZP Piperacillin-Tazobactam, CSL Cefoperazone-Sulbactam, AMK Amikacin, IPM Imipenem, MEM Meropenem. Data on Enteroinvasive E. coli and Coinfection are not shown because of small numbers

${ }^{a}$ For comparison of the resistance percentage among the different pathotypes of Diarrheagenic $E$. coli. STEC does not include, because of too few ${ }^{\mathrm{b}} \mathrm{NA}$, not applicable

norovirus should be monitored. We found that the detection rate of norovirus exhibited a seasonal trend; specifically, detection rates in the winter and spring were significantly higher than those in the summer and autumn, which is consistent with other reports [23, 24]. The current study also revealed the presence of multiple infections, as nearly half of the $\mathrm{HucV}$-positive samples contained multiple infections.

DEC was the most frequently isolated bacterial enteropathogen, supporting the well-documented role of E. coli in diarrheal disease [6]. Our study confirmed that DEC was more frequently isolated from samples from older infants than from samples from younger infants. Quiroga et al. suggested that increased exposure to pathogens after 6 months of age were potentially resulted from the introduction of contaminated foods into the diet [25]. DEC detection rates have been reported to correlate with the seasons [26, 27]. Our study also confirmed that the detection rate of DEC in the summer is higher than in the winter. Many reports have demonstrated the association of EAEC with diarrhea in children. In this study, EAEC was demonstrated to be the most prevalent DEC subtype. The role of EPEC in diarrheal disease has been previously described in several studies, In the current study, we confirmed that EPEC is an important pathogen contributing to diarrhea in children. EPEC infection has been considered a primary disease in infants younger than 2 years of age [27], but in the current study we did not find evidence to confirm this assumption. Most epidemiological studies with data on isolation rates according to age have reported ETEC prevalence rates of $10-20 \%$ among children $<12$ months of age. However, the prevalence of ETEC in Zhejiang province was $1.1 \%$, which is lower than previously the reported rates. The low frequencies of EHEC and EIEC strains in the studied population were, in general, in agreement with other studies performed in different parts of the world $[28,29]$.

Astroviruses are currently considered the third causative agent of gastroenteritis after rotavirus and norovirus 
[30]. In this study, astrovirus was found in $1.6 \%$ of the stool samples, which is a slightly lower percentage than previously reported in China [30]. In the current study, adenoviruses were detected in $2.2 \%$ of the cases, which inconsistent with the rate reported in Tunisian (2.3\%) [31], Australia (3.1\%) [32], and France (3 \%) [33]. Shigella spp. was revealed to play an important role in the diarrhea in all age groups. The frequency of isolation of Shigella spp. was $1.5 \%$ on average, which is markedly lower than the rate reported in other countries [5, 34]. Research has shown that Shigella spp. were more easily detected in older children [34, 35]; however, we did not find such a trend. As reported in the developed regions [36], the dominant pathogen was S. sonnei. Another relevant enteropathogenic bacterium, Vibrio parahaemolyticus, which is a food-borne enteropathogen in coastal areas [34], was found in 29 cases (1.3\%) and was ranked third among bacterial pathogens in this study. Although the incidence of $V$. parahaemolyticus-related diarrhea was not high in the present survey, attention should be paid to the treatment of seafood, which is frequently implicated as a reservoir of marine $V$. parahaemolyticus. Fifteen strains of Salmonella spp. were isolated from diarrheal specimens, which was lower than the number reported in other regions [14]. Additionally, in our study, the presence of Amoeba, one of the most important parasitic pathogens, was significantly associated with diarrhea. Detection rates of Aeromonas hydrophila, Campylobacter jejuni, Plesiomonas shigelloides, Yersinia enterocolitica, Campylobacter spp., Giardia lamblia, and Cryptosporidiosis were all less than $0.5 \%$, which is consistent with other reports $[14,37]$.

Mixed infections are an evolving problem in the epidemiology of diarrhea. With better diagnostic tools, more mixed infections are being identified. The presence of mixed infections complicates the identification of a specific pathogen responsible for the disease and may indicate an additive effect of each pathogen present in a co-infection. In this study, the most common mixed infection included rotavirus and calicivirus, and was present in a total of $55(5.2 \%)$ strains, consistent with research in Spain [13]. We also found 2 cases infected with four types of pathogens, which is rare in other reports.

Traditional antibiotics including trimethoprimsulfamethoxazole, ampicillin, and tetracycline showed low activities against the DEC strains. The resistance to ampicillin was up to $92.4 \%$, higher than that in the neighboring country of Vietnam [38]. Imipenem, meropenem, amikacin, and third-generation cephalosporins are still effective for most bacterial pathogens in the area. However, the use of imipenem and meropenem is not recommended in younger children. As a result, the treatment of choice in cases in which antibiotic therapy is recommended becomes a problem.

\section{Conclusions}

Our results showed that in addition to rotavirus, calicivirus and DEC are important causative agents of childhood diarrhea in Zhejiang province. The high prevalence of rotavirus provides a scientific basis for rotavirus vaccines and other measures to prevent rotavirus infections. This knowledge of the broad spectrum of etiological agents of diarrhea in the participants will help us plan studies on the various aspects of diarrheal diseases in the Chinese population. However, the pathogen spectrum is dynamic, which emphasizes the need to carry out longterm monitoring. Much work remains to be done to decrease the prevalence of diarrhea in children less than 5 years of age in China.

\section{Abbreviations}

CLSI, Clinical Laboratory Standards Institute; DEC, Diarrheagenic E.coli; EAEC, enteroaggregative E. coli; EIEC, enteroinvasive E. coli; EPEC, enteropathogenic E. coli; ESBL, extended-spectrum beta-lactamase; ETEC, enterotoxigenic E. coli; HucV, human calicivirus; PRC, The People's Republic of China; STEC, Shiga toxin-producing E. coli

\section{Acknowledgments}

We thank all the participants for collecting the data and testing the specimens; as well as the Chinese Center for Disease Control and Prevention for support during the development of this project.

\section{Funding}

This study was supported by The National Science and Technology Major Project (Infectious Disease Control and Prevention) (2012ZX10004-210).

\section{Availability of data and materials}

All the data supporting the presented findings are contained within the manuscript.

\section{Author contributions}

All authors participated in interpreting the findings. SZ, XC and DC designed of the study and drafted the manuscript. YC, FY, GX and YX were responsible for sample collection and patient follow-up, DH, YW and WZ carried out pathogen detection. YC was the principal investigator, provided all the facilities necessary to complete this work, and was involved in editing the manuscript. All authors read and approved the final manuscript.

\section{Competing interests}

None of the authors has declared any conflict of interest pertaining to this manuscript.

\section{Consent for publication}

Not applicable.

\section{Ethics and consent to participate}

The study was approved by the Ethical Review Board of the First Affiliated Hospital, College of Medicine, Zhejiang University. Each fecal specimen was collected with the informed consent of the children's parents.

\section{Author details}

${ }^{1}$ State Key Laboratory for Diagnosis and Treatment of Infectious Diseases, Collaborative Innovation Center for Diagnosis and Treatment of Infectious Diseases, The First Affiliated Hospital, College of Medicine, Zhejiang University, No. 79, Qingchun Road, Hangzhou 310003, People's Republic China. ${ }^{2}$ Key Laboratory of Clinical In Vitro Diagnostic Techniques of Zhejiang Province, No. 79, Qingchun Road, Hangzhou 310003, People's Republic China. ${ }^{3}$ Center of Clinical Laboratory, Affiliated Children's Hospital, School of Medicine, Zhejiang University, No. 57, Zhuganxiang Road, Hangzhou 310052, People's Republic China. ${ }^{4}$ Department of Clinical Laboratory, Northern Jiangsu People's Hospital, No. 98, Nantong West Road, Yangzhou 225001, People's Republic China. 
Received: 9 November 2015 Accepted: 7 August 2016 Published online: 20 August 2016

\section{References}

1. Boschi PC, Velebit L, Shibuya K. Estimating child mortality due to diarrhoea in developing countries. Bull World Health Organ. 2008;86(9):710-7.

2. Yan H, Nguyen TA, Phan TG, Okitsu S, Li Y, Ushijima H. Development of RT-multiplex PCR assay for detection of adenovirus and group A and C rotaviruses in diarrheal fecal specimens from children in China. Kansenshogaku Zasshi. 2004;78(8):699-709.

3. Muller D, Greune L, Heusipp G, Karch H, Fruth A, Tschape H, et al. Identification of unconventional intestinal pathogenic Escherichia coli isolates expressing intermediate virulence factor profiles by using a novel single-step multiplex PCR. Appl Environ Microbiol. 2007;73(10):3380-90.

4. Magiorakos AP, Srinivasan A, Carey RB, Carmeli Y, Falagas ME, Giske CG, et al. Multidrug-resistant, extensively drug-resistant and pandrug-resistant bacteria: an international expert proposal for interim standard definitions for acquired resistance. Clin Microbiol Infect. 2011;18(3):268-81.

5. Jafari F, Garcia-Gil LJ, Salmanzadeh-Ahrabi S, Shokrzadeh L, Aslani MM, Pourhoseingholi MA, et al. Diagnosis and prevalence of enteropathogenic bacteria in children less than 5 years of age with acute diarrhea in Tehran children's hospitals. J Infect. 2009;58(1):21-7.

6. Mandomando IM, Macete EV, Ruiz J, Sanz S, Abacassamo F, Valles X, et al. Etiology of diarrhea in children younger than 5 years of age admitted in a rural hospital of southern Mozambique. Am J Trop Med Hyg. 2007;76(3):522-7.

7. Curtis V, Cairncross S, Yonli R. Domestic hygiene and diarrhoea - pinpointing the problem. Trop Med Int Health. 2000;5(1):22-32.

8. Cairncross S, Kolsky PJ. Re: "Water, waste, and well-being: a multicountry study". Am J Epidemiol. 1997;146(4):359-61.

9. Esrey SA. Water, waste, and well-being: a multicountry study. Am J Epidemiol. 1996;143(6):608-23.

10. Rózalski A, Sidorczyk Z, Kotełko K. Potential Virulence Factors of Proteus Bacilli. Microbiol Mol Biol Rev. 1997;61(1):65-89.

11. Binka E, Vermund $\mathrm{SH}$, Armah GE. Rotavirus diarrhea among children less than 5 years of age in urban Ghana. Pediatr Infect Dis J. 2011;30(8):716-8.

12. Parashar UD, Gibson CJ, Bresse JS, Glass RI. Rotavirus and severe childhood diarrhea. Emerg Infect Dis. 2006;12(2):304-6.

13. Sanchez-Fauquier A, Montero V, Colomina J, Gonzalez-Galan V, Aznar J, Aisa ML, et al. Global study of viral diarrhea in hospitalized children in Spain: Results of Structural Surveillance of Viral Gastroenteritis Net Work (VIGESS-net) 2006-2008. J Clin Virol. 2011;52(4):353-8.

14. Rahouma A, Klena JD, Krema Z, Abobker AA, Treesh K, Franka E, et al. Enteric pathogens associated with childhood diarrhea in Tripoli-Libya. Am J Trop Med Hyg. 2011;84(6):886-91.

15. Yang SY, Hwang KP, Wu FT, Wu HS, Hsiung CA, Chang WC, et al. Epidemiology and clinical peculiarities of norovirus and rotavirus infection in hospitalized young children with acute diarrhea in Taiwan, 2009. J Microbiol Immunol Infect. 2010;43(6):506-14.

16. Jin $Y$, Cheng $W X$, Yang $X M$, Jin $M$, Zhang $Q$, Xu ZQ, et al. Viral agents associated with acute gastroenteritis in children hospitalized with diarrhea in Lanzhou. China J Clin Virol. 2009:44(3):238-41.

17. Atchison CJ, Tam CC, Hajat S, van Pelt W, Cowden JM, Lopman BA. Temperature-dependent transmission of rotavirus in Great Britain and The Netherlands. Proc Biol Sci. 2010;277(1683):933-42.

18. Levy K, Hubbard AE, Eisenberg JN. Seasonality of rotavirus disease in the tropics: a systematic review and meta-analysis. Int J Epidemiol. 2009;38(6):1487-96.

19. Koopmans M. Progress in understanding norovirus epidemiology. Curr Opin Infect Dis. 2008;21(5):544-52

20. Zeng $\mathrm{M}, \mathrm{Xu} \mathrm{X}$, Zhu $\mathrm{C}$, Chen J, Zhu Q, Lin S, et al. Clinical and molecular epidemiology of norovirus infection in childhood diarrhea in China. J Med Virol. 2012;84(1):145-51.

21. Podkolzin AT, Fenske EB, Abramycheva NY, Shipulin GA, Sagalova OI, Mazepa VN, et al. Hospital-based surveillance of rotavirus and other viral agents of diarrhea in children and adults in Russia, 2005-2007. J Infect Dis. 2009;200 Suppl 1:S228-33.

22. Fang ZY, Xie HP, Lv HX, Zhang Q, Duan ZJ, Steele D, et al. Investigation of human calicivirus (HuCV) diarrhea among infantile and young children in China, 1999-2005. Bing Du Xue Bao. 2007;23(1):9-15.
23. Mounts AW, Ando T, Koopmans M, Bresee JS, Noel J, Glass RI. Cold weather seasonality of gastroenteritis associated with Norwalk-like viruses. J Infect Dis. 2000;181 Suppl 2:S284-7.

24. Lopman B, Armstrong B, Atchison C, Gray JJ. Host, weather and virological factors drive norovirus epidemiology: time-series analysis of laboratory surveillance data in England and Wales. PLoS One. 2009;4(8):e6671.

25. Quiroga M, Oviedo P, Chinen I, Pegels E, Husulak E, Binztein N, et al. Asymptomatic infections by diarrheagenic Escherichia coli in children from Misiones, Argentina, during the first twenty months of their lives. Rev Inst Med Trop Sao Paulo. 2000;42(1):9-15.

26. Nataro JP, Kaper JB. Diarrheagenic Escherichia coli. Clin Microbiol Rev. 1998;11(1):142-201.

27. Vilchez S, Reyes D, Paniagua M, Bucardo F, Mollby R, Weintraub A. Prevalence of diarrhoeagenic Escherichia coli in children from Leon. Nicaragua J Med Microbiol. 2009;58(Pt 5):630-7.

28. Ochoa TJ, Ecker L, Barletta F, Mispireta ML, Gil Al, Contreras C, et al. Age-related susceptibility to infection with diarrheagenic Escherichia coli among infants from Periurban areas in Lima. Peru Clin Infect Dis. 2009:49(11):1694-702.

29. Torres ME, PMS F, Al E. Etiology of Children's Diarrhea in Montevideo, Uruguay:Associated Pathogens and Unusual Isolates. J Clin Microbiol. 2001:39:2134-9.

30. Liu MQ, Yang BF, Peng JS, Zhou DJ, Tang L, Wang B, et al. Molecular epidemiology of astrovirus infection in infants in Wuhan, China. J Clin Microbiol. 2007:45(4):1308-9.

31. Sdiri-Loulizi K, Gharbi-Khelifi H, de Rougemont A, Hassine M, Chouchane S, Sakly N, et al. Molecular epidemiology of human astrovirus and adenovirus serotypes 40/41 strains related to acute diarrhea in Tunisian children. J Med Virol. 2009;81(11):1895-902

32. Grimwood K, Carzino R, Barnes GL, Bishop RF. Patients with enteric adenovirus gastroenteritis admitted to an Australian pediatric teaching hospital from 1981 to 1992. J Clin Microbiol. 1995;33(1):131-6.

33. Bon F, Fascia P, Dauvergne M, Tenenbaum D, Planson $H$, Petion AM, et al. Prevalence of group A rotavirus, human calicivirus, astrovirus, and adenovirus type 40 and 41 infections among children with acute gastroenteritis in Dijon. France J Clin Microbiol. 1999;37(9):3055-8.

34. Yamashiro T, Nakasone $N$, Higa N, Iwanaga M, Insisiengmay S, Phounane $T$, et al. Etiological study of diarrheal patients in Vientiane, Lao People's Democratic Republic. J Clin Microbiol. 1998;36(8):2195-9.

35. Albert MJ, Faruque AS, Faruque SM, Sack RB, Mahalanabis D. Case-control study of enteropathogens associated with childhood diarrhea in Dhaka. Bangladesh J Clin Microbiol. 1999;37(11):3458-64.

36. Bonfiglio G, Simpore J, Pignatelli S, Musumeci S, Solinas ML. Epidemiology of bacterial resistance in gastro-intestinal pathogens in a tropical area. Int J Antimicrob Agents. 2002;20(5):387-9.

37. Fischer WC, Sack D, Black RE. Etiology of diarrhea in older children, adolescents and adults: a systematic review. PLoS Negl Trop Dis. 2010;4(8):e768.

38. Nguyen TV, Le PV, Le CH, Weintraub A. Antibiotic resistance in diarrheagenic Escherichia coli and Shigella strains isolated from children in Hanoi, Vietnam. Antimicrob Agents Chemother. 2005;49(2):816-9. 